\title{
The Academic and Social Impact of COVID-19 Among College Students: Perspectives from the United States of America, Cameroon, Ghana, and Nigeria
}

\author{
Ernest Kaninjing ${ }^{1}$, Ivette A. Lopez ${ }^{2}$, Che Wankie ${ }^{3}$, Elizabeth O. Akin Odanye ${ }^{4}$, Roland N. Ndip ${ }^{5}$, Yussif \\ M. Dokurugu ${ }^{6}$, Nicholas Tendongfor ${ }^{7}$, Felix Amissah ${ }^{8}$, Shelley White Means ${ }^{9}$, Christopher Paul ${ }^{10}$, Derrick \\ L. Sauls ${ }^{11} \&$ Helene Vilme ${ }^{12}$ \\ ${ }^{1}$ School of Health and Human Performance, Georgia College \& State University, Milledgeville, Georgia, USA \\ ${ }^{2}$ Department of Family and Preventive Medicine, University of Utah, Salt Lake City, Utah, USA AND Utah Area \\ Health Education Centers, University of Utah, Salt Lake City, Utah, USA \\ ${ }^{3}$ Seneca-Solutions, San Diego, California, USA \\ ${ }^{4}$ Department of Clinical Psychology, University of College Hospital, Ibadan, Oyo State, Nigeria \\ ${ }^{5}$ Department of Microbiology and Parasitology, University of Buea, Southwest Region, Cameroon \\ ${ }^{6}$ Institute of Global Health, Inc. Tallahassee, Florida, USA \\ ${ }^{7}$ Department of Public Health and Hygiene, University of Buea, Southwest Region, Cameroon \\ ${ }^{8}$ Department of Pharmaceutical Sciences, Ferris State University, Big Rapids, Michigan, USA \\ ${ }^{9}$ Shelley White-Means, Department of Interprofessional Education, University of Tennessee Health Science Center, \\ Memphis, TN, USA \\ ${ }^{10}$ Department of Public Administration, North Carolina Central University, Durham, North Carolina, USA \\ ${ }^{11}$ Department of Public Health and Exercise Science, Saint Augustine's University, Raleigh, North Carolina, USA \\ ${ }^{12}$ Department of Population Health Sciences, Duke University, Durham, North Carolina, USA \\ Correspondence: Ernest Kaninjing, School of Health and Human Performance, Georgia College \& State University, \\ Milledgeville, GA., 31061, USA. \\ This research was supported by funding from the National Heart, Lung, and Blood Institute under grant [NHLBI \\ 5K12-HL138030-03].
}

Received: September 19, 2021

Accepted: November 3, 2021

Online Published: November 4, 2021

doi:10.5430/ijhe.v11n3p1

URL: https://doi.org/10.5430/ijhe.v11n3p1

\begin{abstract}
The novel coronavirus disease of 2019 (COVID-19) caused disruptions in the delivery of higher education around the globe. To understand how universities and students are dealing with the sudden change from in-person course delivery to online format, this cross-sectional mixed-method study aimed to (a) ascertain the impact of the COVID-19 pandemic on students' ability to access online learning; (b) examine how college students adapted to changes in the learning/teaching environment; and (c) explore the students' perspective on measures that institutions of higher learning could have adopted to ease the abrupt transition to online learning. Results indicate a majority of participants in the US reported access to internet and computers for off-campus learning during the COVID-19 pandemic. A little over half of participants from Africa reported internet access during the COVID-19 pandemic (82\% of participants from Nigeria and $66.7 \%$ from Ghana). Participants from Cameroon reported the lowest percentage of access to online learning at 59.1\%. Participants from Africa reported challenges in adapting to online format due to inadequate access to necessary technological resources such as a reliable internet and computer. Participants identified internal and external resources that could have been adopted to better deal with the transition to online learning. Institutions of higher learning can learn from their initial response to the COVID-19 pandemic to formulate and adjust policies that provide flexibility to effectively transition to online learning while catering to the social, educational and health needs of their students.
\end{abstract}

Keywords: online learning, teaching environment, response to COVID-19, internal and external resources 


\section{Introduction}

\subsection{Problem Statement}

Young adulthood (often defined as persons between the ages of 18 and 30 years) is a complex formative period with the potential to affect the trajectory of an individual's economic security, health, personal development, and well-being. It represents a developmental phase marked by shifts in human physiology, cognitive development, and emotional growth (Commons \& Richards, 1984; Fischer \& Rose, 1994) requiring unique health and social needs. Most individuals who, at this transitional phase have completed high school, are pursuing higher education, or are starting a career, in addition to taking on adult responsibilities (Stroud et al., 2015). Strong personal relationships and healthy habits are formed during this pivotal phase. This period of transition to adulthood increases vulnerability to negative health behaviors such as smoking, vaping, alcohol and recreational drug use, sedentary lifestyle, and risky sexual behaviors (Viner et al., 2015). Moreover, some young adults experience unemployment and homelessness, lack of access to healthcare, and mental health or other chronic health conditions (Stroud et al., 2013).

The challenges of navigating young adulthood were compounded in 2020 by the ripple effects of a pandemic. The novel coronavirus disease of 2019 (COVID-19) emerged as the most common cause of death in the United States of America (Woolf et al., 2020), surpassing cardiovascular disease and cancer which ranked as the leading cause of death in the past decade (Centers for Disease Control \& Prevention, 2020). Globally, young adults faced financial insecurity from the shutdown of economic activities aimed at forestalling the rapid spread of COVID-19. The resulting loss of jobs and income exacerbated food insecurity in developed and developing countries and increased anxiety caused by housing instability (Lederer et al., 2021; The World Bank, 2021; Wolfson \& Leung, 2020). Furthermore, the decision by institutions of higher learning around the globe to switch from in-person to online learning worsened conditions of stress, anxiety, and uncertainty for students and families (Zhai \& Du, 2020). According to UNESCO, on March 31, 2020, schools and universities had closed in 169 countries, affecting $84 \%$ of total enrolled learners (UNESCO, 2021).

\subsection{Literature Review}

Policy measures enacted by the US government to mitigate the economic impact of COVID-19 include the Coronavirus Aid, Relief, and Economic Security (CARES) Act, suspension of student loan payment till January 2022, and allocating funding to institutions of higher learning for emergency financial aid to students (U.S. Department of Education, 2021). Despite these actions, recent studies among university students in the US report increasing levels of food and housing insecurity (Mialki et al., 2021; Soldavini et al., 2021). In addition, many US college students who lost their jobs due to business closures in 2020 are still unemployed in 2021. The unemployment rate for individuals enrolled in college in October 2021 stood at $7.7 \%$ (U.S. Bureau of Labor Statistics, 2021) compared to the national average of 4.8\% (U.S. Bureau of Labor Statistics, 2021). In Africa, where the closure of higher education institutions was more widespread and mitigation efforts became less effective compared to other regions of the world (Organization for Ecoomic Coorporation and Development, 2021), students and institutions of higher learning faced technological and educational infrastructure challenges (Béché, 2020; Stevan Weine, Maarten Bosland, Chandrika Rao, Marcia Edison, Daniel Ansong, Stacey chamberlain, 2021). Students in Africa did not receive any direct financial assistance from their governments to mitigate the economic hardship cause by the shutdown of business and other activities which contributed to psychological distress (Akin-Odanye, E; Kaninjing, E; Ndip, R; Warren, CL; Asuzu, CC; Lopez, I; Muiruri, C \& Vilme, 2021; Awoke et al., 2021). The negative psychosocial impact of the pandemic among college and university students in the US is reported in several recent students which indicate increasing levels of stress and anxiety among this population (Browning et al., 2021; Son et al., 2020; Zhai \& Du, 2020). The current study contributes to the literature and aims at further understanding the academic and social effects of the COVID-19 pandemic on college students in the US and three African countries.

\subsection{Importance of the Problem}

The disruptions in higher education systems by the COVID-19 pandemic amplified existing gaps in access to education, even in developed countries like the US. Online learning requires technological devices like computers, tablets, as well as reliable internet to access course content. In the US, researchers have found that students from low-income backgrounds and ethnic minority communities rely on older technological devices that are inclined to breakdown (Gonzales et al., 2020). Furthermore, researchers found that online learning is most effective when students have consistent access to reliable internet and computers and a conducive study environment, and when teachers are provided training and support to deliver content online (Garcia, Emma, \& Weiss, 2020). Lack of sustainable access to broadband internet has been an obstacle for many students and families in rural communities 
across the US. According to a 2019 Pew Research Center report, rural Americans were 12 percentage points less likely than urban Americans to have home broadband internet (Perrin, 2019). With the partial global shutdown of economic activities and closure of higher education, many students returned home where online learning contents were inaccessible, especially among students in rural areas.

\subsection{Research Objectives}

While institutions of higher education in the US and other developed regions were quicker to adapt to online learning, many universities in Africa were ill-prepared for this abrupt change in learning and teaching. Years of underinvestment in the foundational elements of digital infrastructure such as affordable and quality internet in countries like Cameroon, Nigeria, and Ghana (Bhandari et al., 2020; Mbodiam, 2020; Oye et al., 2011) made the transition to online learning more daunting. To better understand the challenges that college students faced or are facing because of the COVID-19 pandemic, we surveyed college students in four countries: US, Nigeria, Cameroon, and Ghana. The purpose of the study was to (a) ascertain the impact of the COVID-19 pandemic on students' ability to access online learning; (b) examine how college students adapted to changes in the learning/teaching environment; and (c) explore the students' perspectives on measures that institutions of higher learning could have adopted to ease the abrupt transition to online learning.

\section{Methods}

This study used a cross-sectional mixed methods design. Quantitative and qualitative approaches were employed to examine students' ability to cope with a changing learning environment during the COVID-19 pandemic. Ethical approval was obtained from each participating institution prior to study activities.

\subsection{Data Collection}

A questionnaire was administered using Qualtrics between May and October 2020. Study inclusion criteria were students at least 18 years old, currently enrolled at an institution of higher education (university or college) that had transitioned to distance/online learning. Potential participants were sent an e-mail with a link to complete the survey electronically. Once ethical approval was obtained from each of the participating institutions, a convenience sample of college and university students were recruited using universities' intranet systems, classroom announcements, and word of mouth. A standardized text that included information about the study's purpose, eligibility, contact information for the principal investigators, and survey link were used. Once students clicked on the link, they opted into completing the survey by electronically consenting. Students were informed their response to the questionnaire would remain confidential and would not be linked to them in any way. Students were not restricted from sharing the link with other college students. As a result of word of mouth, students from an additional 19 colleges and universities completed the survey to a total of 2035 students in the US and 160 from Cameroon, Ghana, and Nigeria.

The first part of the survey solicited demographic information such as age and gender. The next set of questions inquired about students' university or college, academic classification, full-time or part-time status, and education payment methods. Students were asked about their living arrangement (on or off campus) prior to and since March 2020. Furthermore, students were asked about their current employment status and whether they experienced any hardship in paying for utilities (electricity, water, etc.) and other household amenities. In addition, students were asked about their concerns regarding education and graduation plans. The survey solicited qualitative data by asking participants to describe their experience dealing with COVID-19 and any university or college assistance programs. Moreover, participants were asked about essential resources needed to successfully complete their course work. Results reported in this manuscript are part of a larger study assessing the impact of COVID-19 on college students in the US, Cameroon, Ghana, and Nigeria. The US survey contained 58 questions and was adapted for distribution in Cameroon, Ghana, and Nigeria. The estimated survey completion time was 15 minutes.

\subsection{Quantitative Data Analysis}

Demographic characteristics of students were described using continuous and categorical variables. Means and standard deviations including median and ranges summarized non-normal continuous variables. Categorical variables were described using frequencies and percentages. Participants from the US were categorized into four Census Bureau geographic regions (West, Midwest, South, and Northeast) (US Census Bureau, n.d.) and those from Africa by their respective countries (Cameroon, Ghana, and Nigeria). In the US, only students who reported a college or university geographic location were analyzed. Survey data were analyzed using SAS On Demand for Academics (SAS Institute, Inc., Cary, NC). 


\subsection{Qualitative Data Analysis}

Two researchers with experience in qualitative data analysis independently analyzed data for themes, commonality, and comparison. Line-by-line open coding was used to develop provisional concepts. Axial coding was employed to sort and categorize the data. Each researcher independently developed a code book upon review of transcripts. Emerging themes from the data were further refined after initial coding when the two researchers met to identify trends in the data and to reach consensus on themes. Intercoder reliability of data (Sandelowski, 1995) was addressed by revisiting the transcript when divergent interpretation of text occurred and seeking a common category for that segment of text.

\section{Results}

\subsection{Quantitative}

A total of 2108 students from the US $(n=1,948)$, Cameroon $(n=66)$, Ghana $(n=33)$, and Nigeria $(n=61)$ consented and completed the entire survey. In the US, students were selected from the four US Census Bureau regions: West $(n=80)$, Midwest $(n=915)$, South $(n=859)$, and Northeast $(n=94)$.

Overall, most students were female (72.7\%) and had a median age of 21 years (range $=17-74$ years). Most students reported an undergraduate academic classification (77.5\%) which ranged from first to fifth year, depending on the education system. More students lived off campus (58.6\%) and $88.9 \%$ of them reported having a stable place to live during the pandemic. Most US students (94.5\%) reported private transportation as the preferred mode of commute to campus while most African students (82.6\%) reported public transportation as the preferred mode to commute to campus. Regarding employment status, most African students $(81.9 \%)$ were unemployed while $48.8 \%$ of US students were unemployed at the time of the interview.

Regarding the impact of COVID-19, students were more concerned about delayed graduation (46.4\%) and completion of course work (38.4\%). Most US students (84.6\%) reported having health insurance coverage while only $28.8 \%$ of their African counterparts reported having such coverage. Among US participants, about one-quarter reported having a family member who had contracted COVID-19, and about one-third knew of friends who had been infected. Irrespective of African country, most participants from Africa did not know of a family member or friend who had contracted COVID-19 as of survey completion date (May to August 2020).

Table 1. Socio-demographics of study participants across their location

\begin{tabular}{|c|c|c|c|c|c|c|c|}
\hline \multirow[b]{2}{*}{ Characteristics } & \multicolumn{4}{|c|}{ United States } & \multicolumn{3}{|l|}{ Africa } \\
\hline & $\begin{array}{l}\text { West } \\
(\mathrm{n}=80) \\
\mathrm{n}(\%)\end{array}$ & $\begin{array}{c}\text { Midwest } \\
(\mathrm{n}=915) \\
\text { n (\%) }\end{array}$ & $\begin{array}{l}\text { South } \\
(\mathrm{n}=\mathbf{8 5 9}) \\
\text { n }(\%)\end{array}$ & $\begin{array}{l}\text { Northeast } \\
(\mathrm{n}=94) \\
\mathrm{n}(\%)\end{array}$ & $\begin{array}{l}\text { Cameroon } \\
(\mathrm{n}=66) \\
\mathrm{n}(\%)\end{array}$ & $\begin{array}{c}\text { Ghana } \\
(\mathbf{n}=\mathbf{3 3}) \\
\text { n }(\%)\end{array}$ & $\begin{array}{l}\text { Nigeria } \\
(\mathrm{n}=61) \\
\mathrm{n}(\%)\end{array}$ \\
\hline \multicolumn{8}{|l|}{ Gender } \\
\hline Male & $20(25.3)$ & $204(22.3)$ & $224(26.1)$ & $27(28.7)$ & $20(30.8)$ & $23(79.3)$ & $24(41.4)$ \\
\hline Female & $59(74.7)$ & $687(75.1)$ & $628(73.1)$ & $66(70.2)$ & $45(69.2)$ & $\begin{array}{c}6 \\
(20.7)\end{array}$ & $34(58.6)$ \\
\hline & & & 7 & & & & \\
\hline Other & $0(0.0)$ & $24(2.6)$ & $(0.8)$ & $1(1.1)$ & - & - & - \\
\hline Missing & 1 & 0 & 0 & 0 & 1 & 4 & 3 \\
\hline \multicolumn{8}{|l|}{ Age } \\
\hline Mean (SD) & $20.4(3.00)$ & $24.6(7.9)$ & $22.4(6.5)$ & $19.6(1.3)$ & $24.5(5.2)$ & $\begin{array}{l}26.5 \\
(4.6)\end{array}$ & $\begin{array}{l}27.5 \\
(10.3)\end{array}$ \\
\hline Median (Range) & $\begin{array}{l}20.0 \\
(18-41)\end{array}$ & $22.0(18-66)$ & $\begin{array}{l}20.0 \\
(18-74)\end{array}$ & $\begin{array}{l}19 \\
(18-28)\end{array}$ & $\begin{array}{l}22.5 \\
(19-40)\end{array}$ & $\begin{array}{l}25.0 \\
(19-40)\end{array}$ & $\begin{array}{l}23.0 \\
(17-56)\end{array}$ \\
\hline $\begin{array}{l}\text { Less than } 25 \\
\text { years }\end{array}$ & $75(96.1)$ & $625(68.5)$ & $726(84.5)$ & 93 (98.9) & $46(71.9)$ & $12(37.5)$ & $34(57.6)$ \\
\hline 25 - 34 years & $2(2.6)$ & 197 (21.6) & 95 & $1(1.1)$ & $14(21.9)$ & $15(56.3)$ & $12(20.3)$ \\
\hline
\end{tabular}




$\begin{array}{llccllll}35 \text { years or more } & 1(1.3) & 90(9.9) & 38(4.4) & 0(0.0) & 4(6.2) & 2(6.2) & 13(22.1) \\ \text { Missing } & 2 & 3 & 0 & 0 & 2 & 1 & 2\end{array}$

Education

\begin{tabular}{|c|c|c|c|c|c|c|c|}
\hline Undergraduate & $72(91.1)$ & $640(71.7)$ & 694 (81.9) & 93 (98.9) & $35(53.0)$ & $\begin{array}{l}30 \\
(100.0)\end{array}$ & $39(66.1)$ \\
\hline Graduate & $7(8.9)$ & $253(28.3)$ & $153(18.1)$ & $1(1.1)$ & $31(47.0)$ & $0(0.0)$ & $20(33.9)$ \\
\hline Missing & 1 & 22 & 12 & 0 & 0 & 3 & 2 \\
\hline \multicolumn{8}{|l|}{ College payment* } \\
\hline Family & $60(75.0)$ & $324(35.4)$ & $488(56.8)$ & $80(85.1)$ & $53(83.3)$ & $18(54.5)$ & $41(67.2)$ \\
\hline Scholarship & $36(45.0)$ & $427(46.7)$ & $434(50.5)$ & $29(30.9)$ & - & - & - \\
\hline Student loans & $19(23.8)$ & $515(56.3)$ & $268(31.2)$ & $18(19.1)$ & - & - & - \\
\hline Employer & $2(2.5)$ & $91(9.9)$ & $62(7.2)$ & $1(1.1)$ & - & - & - \\
\hline Personal funds & $23(28.8)$ & $402(43.9)$ & $199(23.2)$ & $8(8.5)$ & $17(25.8)$ & $20(60.6)$ & $20(32.8)$ \\
\hline Other & - & - & - & - & $4(6.1)$ & $3(9.1)$ & $\begin{array}{c}7 \\
(11.5)\end{array}$ \\
\hline
\end{tabular}

Living situation pre

COVID-19

$\begin{array}{lccccccc}\text { On-Campus } & 60(75.9) & 270(31.0) & 404(47.9) & 83(91.2) & 1(1.5) & (12.9) & 23(38.3) \\ \text { Off-Campus } & 19(24.1) & 601(69.0) & 440(52.1) & 8(8.8) & 65(98.5) & 27(87.1) & 37(61.7) \\ \text { Missing } & 1 & 44 & 15 & 3 & 0 & 2 & 1\end{array}$

Living situation during Covid-19

Steady place

$$
74 \text { (92.6) }
$$

$816(89.2)$

$777(90.5)$

$93(98.9) \quad 47(71.2)$

$18(54.5) \quad 50(82.0)$

Worried about losing place

$$
3(3.7)
$$

$86(9.4)$

$69(8.0)$

$1(1.1)$

14 (21.2)

9
$(27.3)$

9

No steady place

Hardship

Yes

$3(3.7)$

$13(1.4)$

$13(1.5)$

$1(1.1)$

$5(7.6)$

6

(18.2)

$2(3.3)$

$\begin{array}{cc}10(14.1) & 260(30.4) \\ 61(85.9) & 594(69.6) \\ 9 & 61\end{array}$

Employed status

Full-time

Part-time

$$
11(13.9)
$$

213 (24.3)

197 (24.2)

$2(2.4) \quad 46(73.0)$

25 (75.8) $28(49.1)$

8

Unemployed

Missing

$16(20.3)$

302 (34.4)

618 (75.8)

$82(97.6)$

17 (27.0)

(24.2)

29 (50.9)

44

10

3

0

4

$$
\begin{array}{rcrrr}
135(16.4) & 9(10.2) & 5(7.6) & \multicolumn{1}{c}{5} \\
257(31.3) & 13(14.8) & 3(4.5) & 1(3.0) & 2(3.3) \\
430(52.3) & 66(75.0) & 58(87.9) & 27(81.8) & 46(75.4)
\end{array}
$$

$257(31.3) \quad 13(14.8) \quad 3(4.5) \quad 1(3.0) \quad 2(3.3)$

$52(65.8) \quad 363(41.3)$

$37 \quad 37 \quad 6$

Reduced work hours due to COVID-19

$$
\text { Yes }
$$$$
13(46.4) \quad 255(46.8)
$$$$
165(38.6)
$$$$
9(33.3) \quad 3(37.5) \quad(16.7)
$$ 


$\begin{array}{lccccccc}\text { No } & 15(53.6) & 290(53.2) & 262(61.4) & 18(66.7) & 5(62.5) & (83.3) & (26.7) \\ \text { Missing } & 0 & 7 & 2 & 1 & & & \\ \text { Campus employment } & & & & & & & \\ & & & & & & 1 & 3 \\ \text { Yes } & 7(25.0) & 104(19.1) & 119(27.9) & 10(37.0) & 1(12.5) & (16.7) & (20.0) \\ & & & & & & 5 & \\ \text { No } & 21(75.0) & 441(80.9) & 308(72.1) & 17(63.0) & 7(87.5) & (83.3) & 12(80.0) \\ \text { Transportation } & & & & & & & \\ \text { Private } & 72(98.6) & 832(95.4) & 784(93.7) & (91.9) & 2(3.4) & (12.0) & 20(33.3) \\ \text { Public } & 1(1.4) & 40(4.6) & 53(6.3) & 7(8.1) & 57(96.6) & 22(88.0) & 40(66.7) \\ \text { Missing } & 7 & 43 & 22 & 7 & 7 & 8 & 1 \\ \text { * Total response percentages exceed } 100 \% . & & & & & \end{array}$

As shown in Figures $1 \mathrm{a} \& 1 \mathrm{~b}$, a majority of students in the US reported access to internet and computers for off-campus learning during the COVID-19 pandemic. A little over half of participants from Africa reported having internet access during the COVID-19 pandemic (82\% of participants from Nigeria and $66.7 \%$ from Ghana). Participants from Cameroon reported the lowest percentage of access to online learning at 59.1\%. In the US, at least $98 \%$ of students in all regions reported access to online learning during the pandemic. While $99 \%$ of US participants reported having computer access for online learning, the percentage for African participants was significantly lower: Cameroon 53\%, Ghana 45.5\%, and Nigeria 59\% (Figures $2 \mathrm{a} \& 2 \mathrm{~b}$ ).
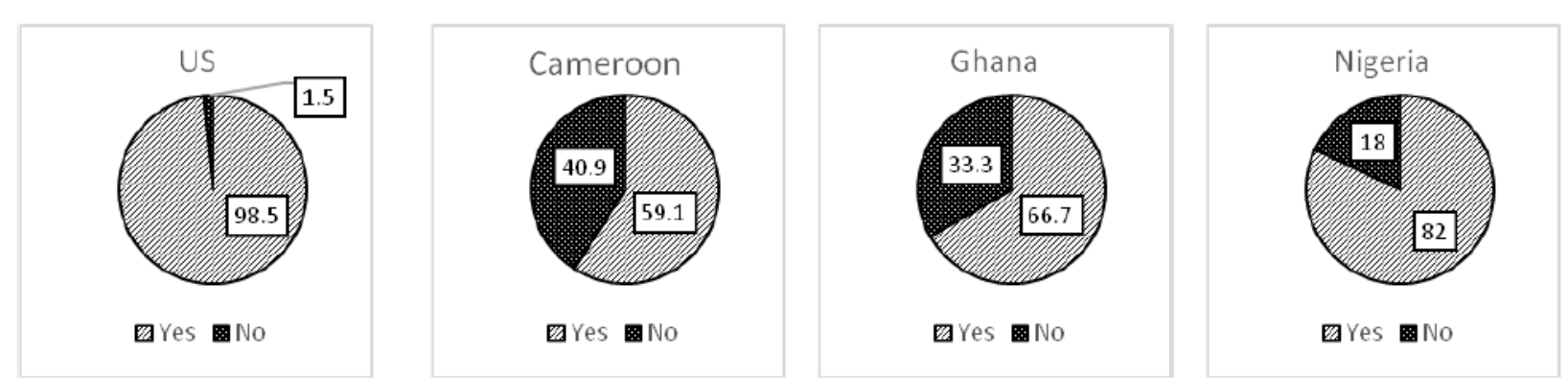

Figure 1a. Frequency of internet access for online learning during COVID-19 among participants in the US and in Africa
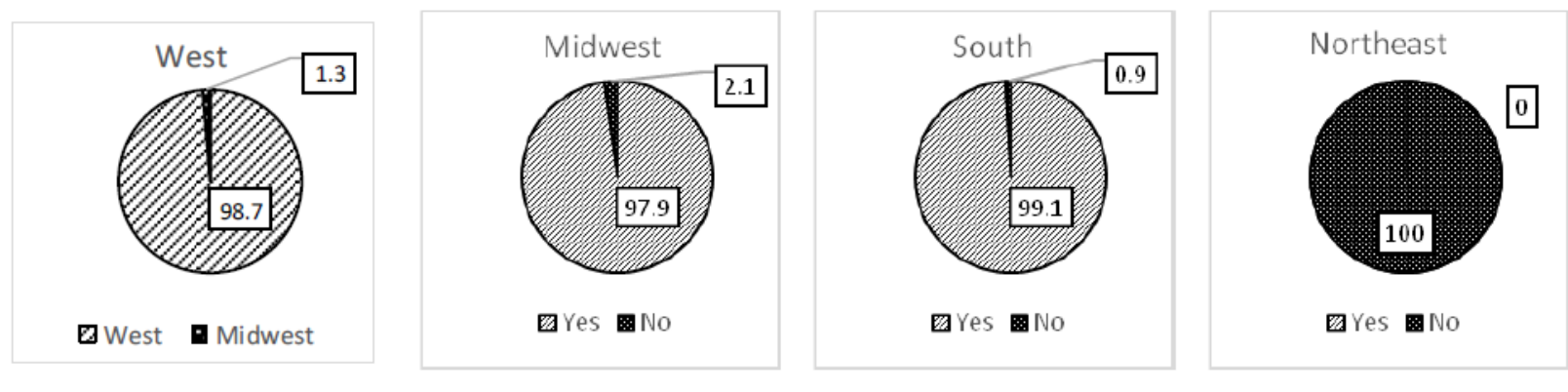

Figure 1b. Frequency of internet access for online learning during COVID-19 among participants in the US, by region 

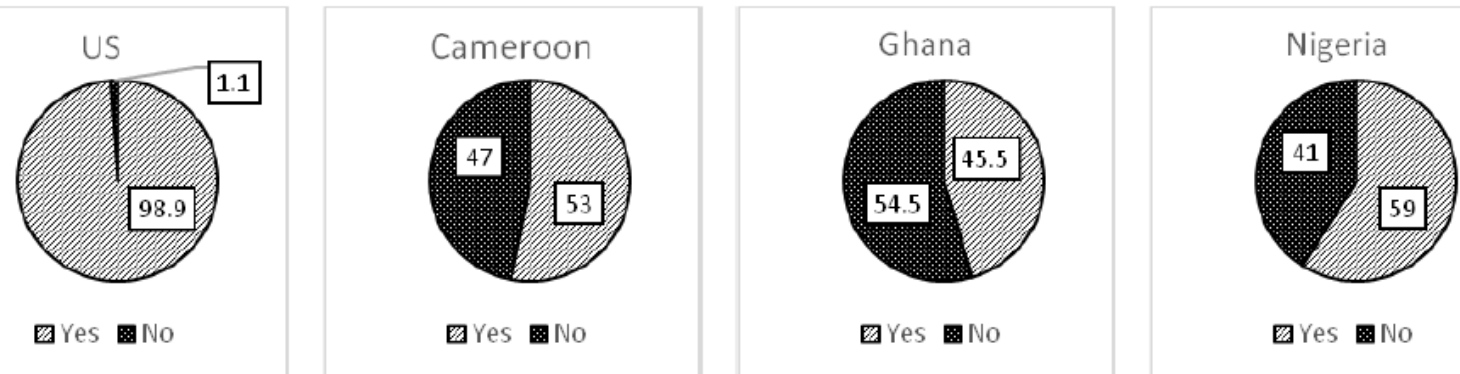

Figure 2a. Frequency of computer access for online learning during COVID-19 among participants in the US and in Africa

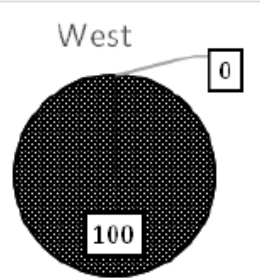

回Yes $\mathrm{BNO}$

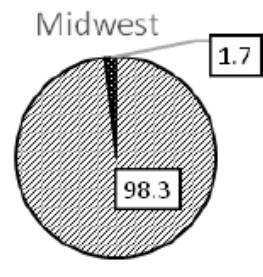

目Yes $\mathrm{No}$

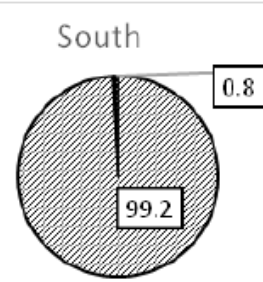

飞Yes $\mathrm{BNO}$

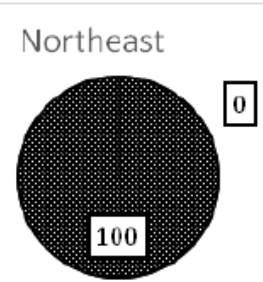

曰

Figure 2b. Frequency of computer access for online learning during COVID-19 among participants in the US, by region

Table 2. Social and health effects of COVID-19 on students

\begin{tabular}{|c|c|c|c|c|c|c|c|}
\hline \multirow[b]{2}{*}{ Characteristics } & \multicolumn{4}{|c|}{ United States } & \multicolumn{3}{|l|}{ Africa } \\
\hline & $\begin{array}{l}\text { West } \\
(\mathbf{n}=80) \\
\text { n }(\%)\end{array}$ & $\begin{array}{c}\text { Midwest } \\
(\mathrm{n}=915) \\
\text { n }(\%)\end{array}$ & $\begin{array}{l}\text { South } \\
(\mathrm{n}=859) \\
\text { n }(\%)\end{array}$ & $\begin{array}{l}\text { Northeast } \\
(\mathrm{n}=94) \\
\mathrm{n}(\%)\end{array}$ & $\begin{array}{l}\text { Cameroon } \\
(\mathrm{n}=66) \\
\mathrm{n}(\%)\end{array}$ & $\begin{array}{c}\text { Ghana } \\
(\mathbf{n}=33) \\
\text { n }(\%)\end{array}$ & $\begin{array}{l}\text { Nigeria } \\
(\mathrm{n}=61) \\
n(\%)\end{array}$ \\
\hline \multicolumn{8}{|l|}{$\begin{array}{l}\text { Effect of COVID-19 } \\
\text { on relationships* }\end{array}$} \\
\hline Parents & $40(50.0)$ & $338(36.9)$ & $291(33.9)$ & $31(33.0)$ & $17(25.8)$ & $13(39.4)$ & $14(23.0)$ \\
\hline Significant other & $24(30.0)$ & $269(29.4)$ & $218(25.4)$ & $15(16.0)$ & $23(34.8)$ & $\begin{array}{c}9 \\
(27.3)\end{array}$ & $14(23.0)$ \\
\hline Friends & $50(62.5)$ & $526(57.5)$ & $454(52.8)$ & $4(4.3)$ & $53(80.3)$ & $20(60.6)$ & $41(67.2)$ \\
\hline \multicolumn{8}{|l|}{$\begin{array}{l}\text { Worries about } \\
\text { COVID-19* }\end{array}$} \\
\hline Delay graduation & $33(41.3)$ & $388(42.4)$ & $373(43.4)$ & $30(31.9)$ & $39(59.1)$ & $21(63.6)$ & $50(81.9)$ \\
\hline $\begin{array}{l}\text { Delay course } \\
\text { completion }\end{array}$ & $42(52.5)$ & $436(47.7)$ & $361(42.0)$ & $44(46.8)$ & $43(61.2)$ & $19(57.6)$ & $29(47.5)$ \\
\hline $\begin{array}{l}\text { Add student } \\
\text { loans }\end{array}$ & $18(22.5)$ & $352(38.5)$ & 205 (23.9) & $12(12.8)$ & $21(31.8)$ & $\begin{array}{c}4 \\
(12.1)\end{array}$ & $11(18.0)$ \\
\hline \multicolumn{8}{|l|}{ Health insurance } \\
\hline Yes & 70 (95.9) & $801(93.0)$ & 767 (95.3) & $89(100.0)$ & $6(9.1)$ & $22(68.7)$ & $22(36.1)$ \\
\hline No & $3(4.1)$ & $60(7.0)$ & $38(4.7)$ & $0(0.0)$ & $60(90.9)$ & $10(31.3)$ & $39(63.9)$ \\
\hline Missing & 7 & 54 & 54 & 5 & 0 & 1 & 0 \\
\hline
\end{tabular}




\begin{tabular}{|c|c|c|c|c|c|c|c|}
\hline $\begin{array}{l}\text { Health insurance } \\
\text { provider* }\end{array}$ & & & & & & & \\
\hline University/College & $17(21.3)$ & $31(3.4)$ & $140(16.3)$ & $13(13.8)$ & $2(3.0)$ & $0(0.0)$ & $9(14.8)$ \\
\hline $\begin{array}{l}\text { Parent/family } \\
\text { member }\end{array}$ & $53(66.3)$ & $555(60.7)$ & $570(66.3)$ & 77 (81.9) & $5(7.6)$ & $16(48.5)$ & $4(6.6)$ \\
\hline Employer & $4(5.0)$ & $124(13.6)$ & $63(7.3)$ & $1(1.1)$ & $0(0.0)$ & $1(3.0)$ & $9(14.8)$ \\
\hline \multicolumn{8}{|l|}{$\begin{array}{l}\text { Preferred medical } \\
\text { treatment location* }\end{array}$} \\
\hline $\begin{array}{l}\text { University/College } \\
\text { clinic }\end{array}$ & $27(33.8)$ & $123(13.4)$ & $296(34.4)$ & $24(25.5)$ & $7(10.6)$ & $14(42.4)$ & $25(41.0)$ \\
\hline $\begin{array}{l}\text { Department of } \\
\text { health clinic }\end{array}$ & $\begin{array}{c}8 \\
(10.0)\end{array}$ & $54(5.9)$ & $71(8.3)$ & $5(5.3)$ & $0(0.0)$ & $0(0.0)$ & $0(0.0)$ \\
\hline $\begin{array}{l}\text { Private } \\
\text { healthcare provider }\end{array}$ & $48(60.0)$ & $593(64.8)$ & $472(54.9)$ & $66(70.2)$ & $16(24.2)$ & $1(3.0)$ & $23(37.7)$ \\
\hline Urgent care & $47(58.7)$ & $474(51.8)$ & 409 (47.6) & $51(54.3)$ & $7(10.6)$ & $0(0.0)$ & $4(6.6)$ \\
\hline $\begin{array}{l}\text { Hospital/Emergency } \\
\text { room }\end{array}$ & $34(3.9)$ & $388(42.4)$ & $309(36.0)$ & $38(40.4)$ & $10(15.2)$ & $\begin{array}{c}4 \\
(12.1)\end{array}$ & $6(9.8)$ \\
\hline \multicolumn{8}{|l|}{$\begin{array}{l}\text { Family contracted } \\
\text { COVID-19 }\end{array}$} \\
\hline Yes & $13(22.0)$ & $205(28.3)$ & $154(22.1)$ & $13(18.3)$ & $1(1.9)$ & $1(3.5)$ & $1(1.7)$ \\
\hline No & $46(78.0)$ & $519(71.7)$ & $542(77.8)$ & $58(81.7)$ & $52(98.1)$ & $28(96.5)$ & $57(98.3)$ \\
\hline Missing & 21 & 191 & 163 & 23 & 13 & 4 & 3 \\
\hline \multicolumn{8}{|l|}{$\begin{array}{l}\text { Friend contracted } \\
\text { COVID-19 }\end{array}$} \\
\hline Yes & $23(31.9)$ & $299(35.0)$ & $333(41.7)$ & $31(34.8)$ & $3(4.6)$ & $2(6.1)$ & $1(1.6)$ \\
\hline No & $27(37.5)$ & $390(45.6)$ & $323(40.4)$ & $30(33.7)$ & $48(72.7)$ & $24(72.7)$ & $48(78.7)$ \\
\hline I don't know & $22(30.6)$ & $166(19.4)$ & 143 (17.9) & $28(31.5)$ & $15(22.7)$ & $\begin{array}{c}7 \\
(21.2)\end{array}$ & $12(19.7)$ \\
\hline Missing & 5 & 60 & 60 & 5 & & & \\
\hline
\end{tabular}

\subsection{Qualitative}

In terms of the impact COVID-19 pandemic is having on students' ability to access online learning, some participants reported that shifting to online learning was difficult due to inadequate access to necessary technological resources such as a reliable internet and computer.

Stable internet connection:

"I may be lucky because I have a steady job. Most of my colleagues do not; accessing the internet for online classes is therefore very challenging for them. There is also the problem of mastery of the zoom classroom access and use. Some training will do great help both for us students and some lecturers using the classroom."- A 39-year-old male graduate student from Cameroon.

"Provide us reliable internet and reduce our fees."- A 23-year-old male undergraduate from Ghana.

"I wish we have free access to or subsidized internet cost, as well as steady internet connections to complete our classes successfully."- A 45-year-old female graduate student from Nigeria. 
"Reliable internet (sometimes zoom is overwhelming for internet connection and it makes attending class more difficult)."- A 19-year-old female sophomore from the Northeast, US.

"More reliable internet access than I currently have - mine is very sporadic and often out for hours or days at a time." - A 25-year-old senior from the South, US.

Computer or electronic device:

"The online work is highly difficult for me because there is no laptop on me to do my online work." - A 23-year-old male undergraduate student from Ghana.

“A laptop to use for my assignments, schoolwork and project, as the one I was using got bad."- A 29-year-old graduate student from Nigeria.

"A reliable computer, constant internet connection, organized class schedule, reliable lecturers." - A 23-year-old male undergraduate student from Cameroon.

"An ipad for physics would be very helpful, I do not have one." - A 20-year-old female sophomore from the West, US.

Additionally, participants enumerated specific resources that could ease their transition and adaptation to an online learning environment. These resources could be categorized into objects, equipment/resources, environment, social support, and reliance on institutional resources as depicted in Table 3.

Table 3. Necessary Resources Identified by Students

\begin{tabular}{ll}
\hline Objects & Equipment/Resources \\
\hline Laptop computer, electronic tablet & Reliable internet access \\
Textbooks, notebooks & University-issued subscription to online journals \\
Recorded video lectures & Money to pay for tuition and fees, food items \\
Printer, scanner, camera & Financial resources to pay for internet access \\
Phone & Constant electricity supply \\
Environment & Social Support \\
Quiet environment & Collaborate in person with classmates \\
Interaction with professor & Understanding and flexibility from faculty in \\
Office hour & accommodating needs of students studying at home \\
Accessible teaching assistants & Dependency on Institutional Resources \\
& Abrupt termination of accustomed resources \\
Access to university library & (wifi, printers, computers, library, etc). \\
& Office hours/Interaction with professors \\
Accessible Teaching Assistants & Access to university library \\
\hline
\end{tabular}

Furthermore, due to the shift by universities and colleges to online learning, students had to adapt to a new learning/teaching environment which some participants felt were not equivalent in quality to in-person learning.

"To be frank, online learning is nothing like in-person classes. The learning environment is not as friendly or engaging, and future terms affected by COVID should acknowledge this."'- A 21-year-old male junior from the South, US.

"The academic versions of online learning quite frankly suck, and they don't compare with the education one receives from a physical location class." - A 47-year-old male senior from the Midwest, US.

"Professors and students seem to have had a poor transition to online, I feel like class discussion is now nonexistent and out of class work has grown exponentially." - A 21-year-old male junior from the Midwest, US.

Besides concerns about the quality of online learning, some participants highlighted challenges with the online study environment at home and their struggle to stay motivated. 
"I along with many other students find it difficult to be motivated to complete my work when it is online. It also makes it hard to keep a routine and feel involved on campus." - A 20-year-old junior from the South, US.

"The atmosphere at home isn't suitable for remote learning. There are too many distractions. I really miss attending physical classes. I feel that there is a degree of accountability that is lost when I have to make do with remote learning." - An 18-year-old female freshman from the South, US.

"Online learning at the university level for stem majors is extremely difficult. There are so many distractions at home that make staying focused on a computer screen for many hours a day very hard. Not having the ability to watch a teacher work through a problem in person makes learning material so much more difficult."'- A 20-year-old male junior from the South, US.

"Not really the best. The worst experience ever. We weren't allowed to go out. So I missed church services. Then online learning came, it was so difficult with no data and poor network." - A 22-year-old female graduate student in Cameroon.

When asked about measures that could have been adopted by their institution to better handle disruptions caused by COVID-19, student's views were classified under internal and external resources. Internal resources included items that can enhance an individual's capacity to deal with behavioral factors amid the COVID-19 pandemic, while external resources were out of the individual's control. Below are some examples of internal and external resources that participants wished their university or college could provide to help deal with the COVID-19 pandemic.

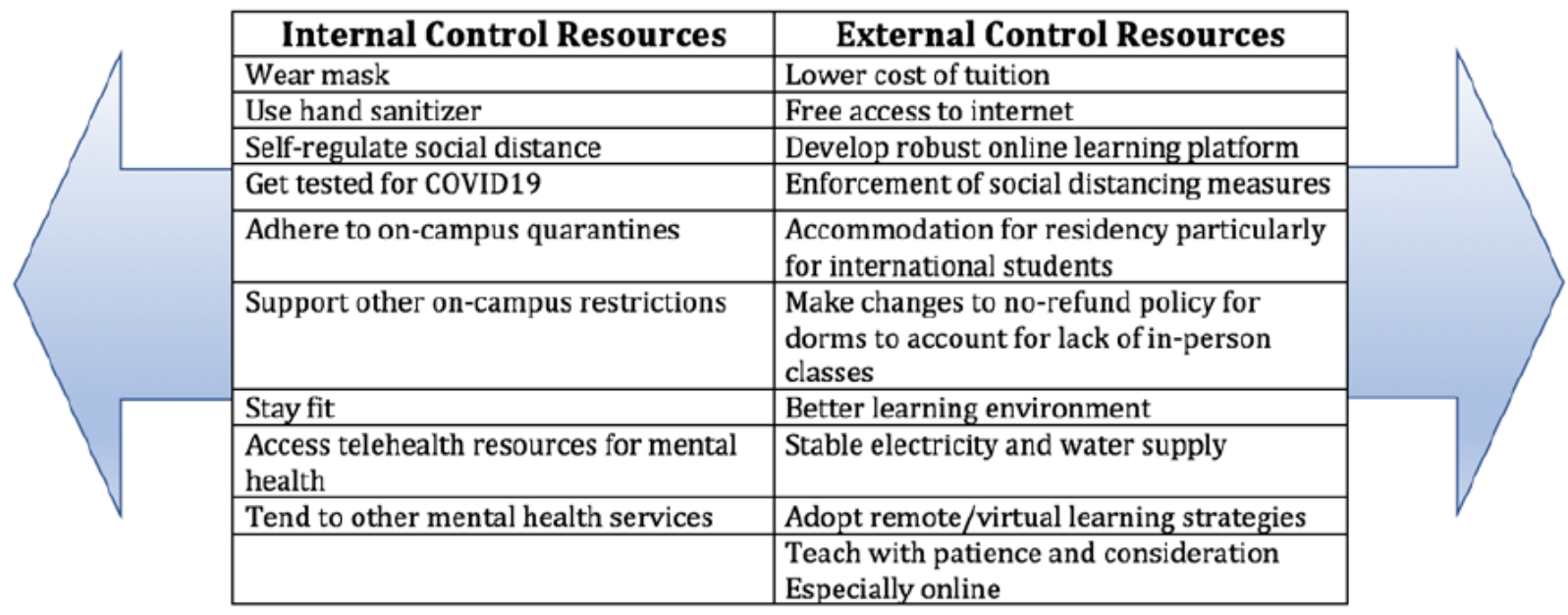

Figure 3. Internal vs External Locus of Control

Selected INTERNAL Illustrative Participant Quotes

"Provide masks and hand sanitizer across campus, enforce social distancing, shut down bars. "A 19-year-old female sophomore student from the South, US.

"Free screening students for COVID 19 and ensure social distancing and wearing of facemasks because up till now some students walk on campus without face masks." A 22- year-old female undergraduate participant from Cameroon.

"I think making masks mandatory will help ease some of my concerns. I also think we should provide free testing to students before they return to campus, and routine testing every 14 days. Quarantine options should be provided. Provide more details about RSO restrictions and guidelines. ”A 20-year-old female Junior student from the South, US.

"To provide social and educational support to students as I believe everyone is under duress due to the pandemic. ”A 42-year-old female graduate student from the South, US.

"Make fitness facilities open and available. That is how I let off steam. That is how I mentally take care of myself. "A 19-year-old female sophomore from the Midwest, US. 
“Offer Psychologist, Wellness Check-in via Email.”A 27-year-old female medical student from the South, US.

"I think the psychological aspect of COVID-19 was largely overlooked. My mental health, and I know many of my peers' mental health, is and has been greatly affected by this pandemic for a multitude of reasons. "A 21-year-old female junior from the South, US.

Selected EXTERNAL Illustrative Participant Quotes

“Start online classes soonest cos COVID doesn't seem like it's going anytime soon. Reduce fees and slash unnecessary payments. ” A 19-year-old female undergraduate participant from Nigeria.

"I wish we have free access to or subsidized internet cost, as well as steady internet connections to complete our classes successfully.” A 39-year-old male graduate studentfrom Cameroon.

"Put a good system in place for online class, and take it serious. In addition give homework and consider them an CA and exams. "A 30-year-old male graduate student from Cameroon.

"My university can change its teaching style to better fit virtual learning." A 27-year-old female graduate student from the South, US.

"I have not been able to independently complete ADLs outside of my home like grocery shopping due to the uncontrolled spread and insufficient public safety enforcement and public feelings. "A 23-year-old female graduate student from Midwest. US.

"Support international students. 1) don't leave us as an afterthought when making policy (eg the housing disaster where many int'l students were kicked off campus - that was extremely upsetting, stressful, and potentially dangerous) 2) financial support for students who had to leave/have nowhere else to go 3) admin support for visa issues - both work AND study." A 21-year-old female senior, international student originally from Thailand.

"And in terms of schooling too they should help me with my school fees and hostel fees." A 23-year-old female undergraduate participant from Ghana.

"Provide housing alternatives in the event that on-campus housing closes down due to pandemic-related reasons. "A 23-year-old female graduate student from the South, US.

In the experience of the participants, mostly internally controlled items indicate that frustrations at the individual level are internally controlled, while those that are related to necessary institutional support are beyond individual behaviors and are externally controlled. Even choices regarding accessing mental health help could be placed in the external control side, due to lack of institutional support.

\section{Discussion}

This study set out to examine the impact of COVID-19 on students' access to online learning, explore students' experiences in adjusting to online learning, and ascertain what measures could have facilitated a smoother transition to online learning from the students' perspective. In terms of access to online learning, our study found $99 \%$ of US participants reported internet and computer access among all four geographic regions. In Africa, participants reported higher levels of online access but lower levels of computer access. The lack of computer access for online learning among participants in Africa was high in both our quantitative and qualitative findings. According to the 2018 International Telecommunications Union, only $16.9 \%$ of households in Cameroon had a computer, and in Nigeria, only $8.1 \%$ of households had access to a computer (International Telecommunication Union, 2019). In Ghana, the percentage of households with computer access stood at 7.9 in 2019 (National Communications Authority, 2020). The very low levels of computer access among African students impeded their ability to adopt to online learning. Some students used their handheld electronic devices to access online course content, which is an inefficient method to browse or download content material in a typical college course. The high cost of online courses was of concern with students asking for lower tuition, particularly given the economic hardship brought about by the shutdown of economic activities.

The psychosocial impact of COVID-19 on study participants was prominent in both the qualitative and quantitative data. Across three of the four regions of the US and all three countries in Africa, over 50\% of participants reported COVID-19 impacting their relationships with friends. This was largely due to social distancing measures imposed to limit community spread of the disease. These measures contributed to anxiety and stress reported by participants in the US and in Africa. Stress was even more pronounced among university seniors facing uncertainty about completing academic studies and dim prospects for employment following the shutdown of economic activities. 
Looking at these findings through the theoretical framework of Locus of Control, most students perceived that the needed resources identified were on the external control pole. Individuals tend to believe events are outside their control when they find themselves with an external locus of control. This perceived lack of control may be causing the anxiety and depression expressed by many students. This is mirrored by the perception of many in the wider US population who feel deprived of decision-making opportunities, having to abide by decisions made by others. This out-of-control situation may be causing or compounding already stressed students.

\section{Recommendations}

As institutions of higher learning across the globe continue to formulate and modify their public health emergency response policies, some recommendations could be made from findings of this study. As noted previously under external control resources, the need for a robust online learning platform was the dominant theme from participants in Africa. Investment in broadband infrastructure by universities in Nigeria, Ghana and Cameroon is inadequate, and reliable supply of electricity is not guaranteed (Egoeze et al., 2014; Oye et al., 2011). To improve their capacity to effectively serve their students, universities in the three African countries should consider investing in digital infrastructure and support as well as training of faculty and staff in online content development and delivery.

Adopting learning-friendly policies that accommodate students' needs could alleviate some of the worry and anxiety expressed by participants. For example, providing students with reliable access to the internet, enough time and the resources to get their work done will address some of the dissatisfaction expressed by participants from Ghana, Nigeria, and Cameroon about their instructors' responses to online learning. In fact, students could be involved and consulted as policies are developed. Contingencies on behalf of students can be the product of collaborations between institutions of higher learning and technological industries. Innovation in remote education is a win for industries supplying equipment and know-how, and a win for students and institutions of higher learning.

In general, providing choices for students to make would be well received. Choices between online, in-person or hybrid learning should be provided. Evaluation of courses should be considered closely to allow for modifications that can empower students into a new normality.

\subsection{Limitations}

This study used a cross-sectional mixed-methods design which did not permit an assessment of any causal relationships. Although our findings describe the challenges students faced during the COVID-19 pandemic, the educational institutions whose students were surveyed in the study are not representative of all universities and colleges in their respective countries. Therefore, inferences and generalizations should be made with caution. In addition, the small sample from Cameroon, Nigeria and Ghana preclude from generalizing findings in this study but reinforces the challenges encountered by some students during the COVID-19 pandemic. Moreover, the cross-sectional approach captures the views and opinions of students at the onset of the pandemic (May to October 2020), and students' views may have changed as the pandemic wore on and students became more informed. As is the case with self-reported questionnaires, participants' responses could not be verified or validated. The response rate was not determined in this study given that the number of survey invitations were not tracked by each university or college in the study. There is a potential of selection bias in that students who had no access to the internet could not complete the survey. Even though the COVID-19 pandemic has been detrimental to students' achievement, the survey did not ascertain whether students experienced hardship prior to the COVID-19 pandemic and whether existing hardships continued or worsened during the pandemic. Conversely, the long-term impact of COVID-19 on students cannot yet be determined but is an area for future research.

\section{Conclusion}

Emergency public health events are likely to occur in the future, causing disruptions in teaching and learning modalities across the globe. Institutions of higher learning can learn from their initial response to the COVID-19 pandemic to formulate and adjust policies that provide flexibility to an effective transition to online learning while catering to the social, educational and health needs of their students. Policies focused on planning, understanding, and seeking strategies to address the needs of the students will contribute to a more successful adoption of online learning.

\section{References}

Akin-Odanye, E., Kaninjing, E., Ndip, R., Warren, C. L., Asuzu, C. C., Lopez, I., Muiruri, C., \& Vilme, H. (2021). Psychosocial Impact of COVID-19 on Students at Institutions of Higher Learning. European Journal of Education Studies, 8(6). https://doi.org/10.46827/EJES.V8I6.3770 
Awoke, M., Mamo, G., Abdu, S., \& Terefe, B. (2021). Perceived Stress and Coping Strategies Among Undergraduate Health Science Students of Jimma University Amid the COVID-19 Outbreak: Online Cross-Sectional Survey. Frontiers in Psychology, 12. https://doi.org/10.3389/fpsyg.2021.639955

Béché, E. (2020). Cameroonian responses to COVID-19 in the education sector: Exposing an inadequate education system. International Review of Education. https://doi.org/10.1007/s11159-020-09870-x

Bhandari, S., Shaktawat, A. S., Patel, B., Dube, A., Kakkar, S., Tak, A., Gupta, J., \& Rankawat, G. (2020). The sequel to COVID-19: the antithesis to life. Journal of Ideas in Health. https://doi.org/10.47108/jidhealth.vol3.issspecial1.69

Browning, M. H. E. M., Larson, L. R., Sharaievska, I., Rigolon, A., McAnirlin, O., Mullenbach, L., Cloutier, S., Vu, T. M., Thomsen, J., Reigner, N., Metcalf, E. C., D’Antonio, A., Helbich, M., Bratman, G. N., \& Alvarez, H. O. (2021). Psychological impacts from COVID-19 among university students: Risk factors across seven states in the United States. PLOS ONE, 16(1). https://doi.org/10.1371/journal.pone.0245327

Centers for Disease Control \& Prevention. (2020). Leading Causes of Death. National Center for Health Statistics. https://www.cdc.gov/nchs/fastats/leading-causes-of-death.htm

Commons, M. L., \& Richards, F. A. (1984). Applying the general stage model. In Beyond formal operations: Late adolescent and adult cognitive development.

Egoeze, F., Misra, S., Akman, I., \& Colomo-Palacios, R. (2014). An evaluation of ICT infrastructure and application in Nigeria universities. Acta Polytechnica Hungarica.

Fischer, K. W., \& Rose, S. P. (1994). Dynamic development of coordination of components in brain and behavior: A framework for theory and research. In Human behavior and the developing brain.

Garcia, E., \& Weiss, E. (2020). COVID-19 and student performance, equity, and U.S. education policy. https://files.epi.org/pdf/205622.pdf

Gonzales, A. L., McCrory Calarco, J., \& Lynch, T. (2020). Technology Problems and Student Achievement Gaps: A Validation and Extension of the Technology Maintenance Construct. Communication Research. https://doi.org/10.1177/0093650218796366

International Telecommunication Union. (2019). Measuring digital development: Facts and figures. In ITUPublications.

Lederer, A. M., Hoban, M. T., Lipson, S. K., Zhou, S., \& Eisenberg, D. (2021). More Than Inconvenienced: The Unique Needs of U.S. College Students During the COVID-19 Pandemic. Health Education and Behavior. https://doi.org/10.1177/1090198120969372

Mbodiam, B. (2020). Cameroon's internet penetration rate reaches $30 \%$ in 2020, thanks to the arrival of 570,000 new internet users. Investir $\mathrm{Au}$ Cameroun. https://www.investiraucameroun.com/economie/2402-14084-le-taux-de-penetration-de-1-internet-au-camerounatteint-30-en-2020-grace-a-1-arrivee-de-570-000-nouveaux-internautes

Mialki, K., House, L. A., Mathews, A. E., \& Shelnutt, K. P. (2021). Covid-19 and College Students: Food Security Status before and after the Onset of a Pandemic. Nutrients, 13(2), 1-13. https://doi.org/10.3390/NU13020628

National Communications Authority, G. S. S. (2020). Household survey on ICT in Ghana (abridged). National survey on ICT access, usage, skills and digital divide in Ghana.

Organization for Ecoomic Coorporation and Development. (2021). COVID-19 impact on higher education in Africa Development

Matters.

https://oecd-development-matters.org/2021/05/19/covid-19-impact-on-higher-education-in-africa/

Oye, N., Salleh, M., \& Iahad, N. (2011). Challenges of E-Learing in Nigerian University Education Based on the Experience of Developed Countries. International Journal of Managing Information Technology. https://doi.org/10.5121/ijmit.2011.3204

Perrin, A. (2019). Digital gap between rural and nonrural America persists. Pew Research Center. https://www.pewresearch.org/fact-tank/2019/05/31/digital-gap-between-rural-and-nonrural-america-persists/

Sandelowski, M. (1995). Qualitative analysis: What it is and how to begin. Research in Nursing \& Health, 18(4), 371-375. https://doi.org/10.1002/nur.4770180411

Soldavini, J., Andrew, H., \& Berner, M. (2021). Characteristics associated with changes in food security status 
among college students during the COVID-19 pandemic. Translational Behavioral Medicine, 11(2), 295-304. https://doi.org/10.1093/TBM/IBAA110

Son, C., Hegde, S., Smith, A., Wang, X., \& Sasangohar, F. (2020). Effects of COVID-19 on College Students' Mental Health in the United States: Interview Survey Study. Journal of Medical Internet Research, 22(9). https://doi.org/10.2196/21279

Stevan Weine, Maarten Bosland, Chandrika Rao, Marcia Edison, Daniel Ansong, \& Stacey chamberlain, A. B. (2021). Global Health Education Amidst COVID-19: Disruptionsnd and Opportunities. Annals of Global Health, 87(1), 1-6. https://doi.org/10.5334/AOGH.3088

Stroud, C., Mainero, T., \& Olson, S. (2013). Improving the Health, Safety, and Well-Being of Young Adults: Workshop Summary. In National Academy Press.

Stroud, C., Walker, L. R., Davis, M., \& Irwin, C. E. (2015). Investing in the health and well-being of young adults. Journal of Adolescent Health. https://doi.org/10.1016/j.jadohealth.2014.11.012

The World Bank. (2021). Food Security and COVID-19. https://www.worldbank.org/en/topic/agriculture/brief/food-security-and-covid-19

U.S. Bureau of Labor Statistics. (2021). BLS Data Viewer. In Unemployment Rate. https://beta.bls.gov/dataViewer/view/timeseries/LNS14000000\%0Ahttps://beta.bls.gov/dataViewer/view/timese ries/LNU02070192

U.S. Department of Education. (2021). CARES Act: Higher Education Emergency Relief Fund.

UNESCO. (2021). Education: From disruption to recovery. https://en.unesco.org/covid19/educationresponse

US Census Bureau. (n.d.). 2018 FIPS Codes. Retrieved July 31, 2021, from https://www.census.gov/geographies/reference-files/2018/demo/popest/2018-fips.html

Viner, R. M., Ross, D., Hardy, R., Kuh, D., Power, C., Johnson, A., Wellings, K., McCambridge, J., Cole, T. J., Kelly, Y., \& Batty, G. D. (2015). Life course epidemiology: Recognising the importance of adolescence. In Journal of Epidemiology and Community Health. https://doi.org/10.1136/jech-2014-205300

Wolfson, J. A., \& Leung, C. W. (2020). Food Insecurity During COVID-19: An Acute Crisis With Long-Term Health Implications. American Journal of Public Health, 110(12), 1763-1765. https://doi.org/10.2105/AJPH.2020.305953

Woolf, S. H., Chapman, D. A., \& Lee, J. H. (2020). COVID-19 as the Leading Cause of Death in the United States. In JAMA - Journal of the American Medical Association. https://doi.org/10.1001/jama.2020.24865

Zhai, Y., \& Du, X. (2020). Addressing collegiate mental health amid COVID-19 pandemic. In Psychiatry Research. https://doi.org/10.1016/j.psychres.2020.113003

\section{Copyrights}

Copyright for this article is retained by the author(s), with first publication rights granted to the journal.

This is an open-access article distributed under the terms and conditions of the Creative Commons Attribution license (http://creativecommons.org/licenses/by/4.0/). 